\title{
Hypercapnia intensifies cerebral hypoxia via increasing cerebral oxygen extraction ratio: implication in neuroinflammation in hypoxemic adult rats
}

\section{Hong-Guang Ding}

Guangdong Provincial People's Hospital and Guangdong Academy of Medical Sciences

\section{Xu-Sheng Li}

Guangdong Provincial People's Hospital and Guangdong Academy of Meidcal Sciences

\section{Xin-Qiang Liu}

Guangdong Provincial People's Hospital and Guangdong Academy of Medical Sciences

\section{Kang-Rong Wang}

Southern Medical University

\section{Ya Li}

South China University of Technology

\section{Miao-Yun Wen}

Guangdong Provincial People's Hospital and Guangdong Academy of Medical Sciences

\section{Hongke Zeng ( $\nabla$ zenghongke@vip.163.com )}

Guangdong Provincial People's Hospital and Guangdong Academy of Medical Sciences https://orcid.org/0000-0003-3243-4199

\section{Research}

Keywords: acute respiratory distress syndrome, hypercapnia, cerebral oxygen extraction ratio, neuroinflammation, microglia

Posted Date: November 12th, 2019

DOI: https://doi.org/10.21203/rs.2.17155/v1

License: (c) (1) This work is licensed under a Creative Commons Attribution 4.0 International License. Read Full License 


\section{Abstract}

Background: Hypoxemia is a typical symptom of acute respiratory distress syndrome (ARDS). Ventilations are always needed for correcting hypoxemia. To avoid pulmonary morbidity, the low tidal volume ventilation is often applied. The ventilation strategy will certainly result in hypercapnia. Our previous study found that hypercapnia increase neuronal death and aggravate the cognitive function of hypoxic adult rats. However, the underlying mechanism has remained unclear. This study aimed to explore whether hypercapnia would induce neuroinflammation through increasing cerebral oxygen extraction ratio in adult rats with hypoxemia.

Methods: Cerebral oxygen extraction ratio (CERO2), partial pressure of brain tissue oxygen (PbtO2), and reactive oxygen species (ROS) production in brain tissue in a rat model of hypercapnia/hypoxemia were evaluated. Along with this, the oxygen consumption rate (OCR) and ROS production of BV-2 microglial cells were evaluated after $15 \% \mathrm{CO} 2 / 0.2 \% 02$ treatment. The protein expression level of caspase- 1 and IL$1 \beta$ in microglia cells were detected before and after application of a ROS scavenger in vivo and in vitro.

Results: Pbt02 level was elevated by hypercapnia in the hypoxemic rats in the first $1.5 \mathrm{~h}$, but it was significantly decreased $2 \mathrm{~h}$ after ventilation. This was further evident by the increased levels of CERO2 at $3 \mathrm{~h}$ after ventilation. Besides, a high concentration of $\mathrm{CO} 2$ treatment could increase the levels of OCR in hypoxic BV-2 microglial cells in vitro. Hypercapnia markedly increased the production of ROS and the expression of caspase-1 and IL-1 $\beta$ in hypoxia-activated microglia both in vivo and in vitro. Pharmacological scavenging ROS inhibited the NLRP3 inflammasome activation and expression of IL-1 $\beta$.

Conclusion: Hypercapnia-intensified cerebral hypoxia via increasing cerebral oxygen extraction ratio may induce ROS overproduction, activate the NLRP3 inflammasome and enhance IL-1 $\beta$ release in hypoxiaactivated microglia.

\section{Background}

Acute respiratory distress syndrome (ARDS) is a common critical disease in intensive care units (ICUs) $[1,2]$. Persistent hypoxemia is a typical symptom of ARDS. Ventilations are always needed to correct hypoxemia[3,4] To avoid or reduce pulmonary morbidity, the low tidal volume ventilation is often applied[5,6]. The ventilation strategy will certainly result in hypercapnia, which is called "permissive hypercapnia" $[7,8]$. Our previous study found that increase neuronal death and aggravate the cognitive function of hypoxic adult rats[9]. However, the underlying mechanism has remained unclear.

The NLRP3 inflammasome is widely expressed in microglia, which plays an important role in initiating neuroinflammation[10-12]. To exert its functions, the NLRP3 inflammasome requires to be activated. The NLRP3 inflammasome can be triggered by an array of stimuli, including ATP, potassium efflux, heme, urate, and reactive oxygen species (ROS)[13-17]. Activation of the NLRP3 inflammasome can upregulate

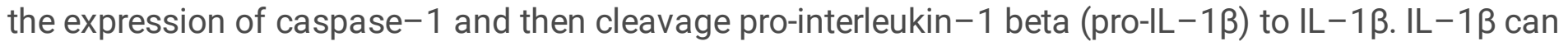
promote the central nervous system cascade inflammatory response and lead to aggravation of neuronal 
injury[18,19]. When the levels of oxygen fall, hypoxia would inhibit the respiratory chain and induce ROS production[20-22]. It remains to be ascertained whether hypercapnia would activate the NLRP3 inflammasome through increasing ROS overproduction in hypoxia-activated microglia.

Neurons and neuronal functions are highly sensitive to hypoxia. Under hypoxic conditions, the oxidative metabolism rate of the brain would decrease to prevent the partial pressure of brain tissue oxygen $\left(\mathrm{PbtO}_{2}\right)$ from dropping to a very low level[23,24]. However, it is still unknown whether hypercapnia would exert any effects on oxidative metabolism of the hypoxic brain.

In the present study, we hypothesized that hypercapnia may induce the NLRP3 inflammasome activation in microglia via intensifying cerebral hypoxia in adult rats with hypoxemia. It was surmised that hypercapnia might exert its effect through increasing $\mathrm{CERO}_{2}$, decreasing $\mathrm{PbtO}_{2}$, and inducing ROS production, which ultimately would activate the NLRP3 inflammasome in microglia and enhance the release of $\mathrm{IL}-1 \beta$.

\section{Methods}

\section{Animals and experimental groups}

Male adult Sprague-Dawley (SD) rats $(n=128)$ aged about 3 months and weighing approximately 220 to $250 \mathrm{~g}$ were randomly divided into five groups: sham-operated group (abbreviated Sham group), Hypercapnia group, Hypoxemia group, Hypercapnia + Hypoxemia group (abbreviated HH group), Hypercapnia + Hypoxemia + N-Acetyl-L-cysteine group (abbreviated HH + NAC group).

\section{The rat model of hypercapnia/hypoxemia}

All rats were fasted with access to water overnight before the experiments. The rat model of hypercapnia/hypoxemia was established as described in our previous study[9]. Briefly, the rats were anesthetized with pentobarbital sodium followed by mechanical ventilation. The tidal volume $(9 \mathrm{ml} / \mathrm{kg}$ body weight), respiratory rate (45 breaths/min), and inspiratory to expiratory ratio (1:1) were fixed. Sham group was exposed to the air. Hypercapnia group was exposed to $5 \% \mathrm{CO}_{2}$ to maintain $\mathrm{pH}$ of arterial blood at $7.20-7.25$. Hypoxemia group was exposed to $16 \% \mathrm{O}_{2}$ to maintain partial pressure of artery blood oxygen $\left(\mathrm{PaO}_{2}\right)$ at $55-60 \mathrm{mmHg}$. $\mathrm{HH}$ group was exposed to $16 \% \mathrm{O}_{2}$ mixing with $5 \% \mathrm{CO}_{2}$ to maintain $\mathrm{PaO}_{2}$ at $55-60 \mathrm{mmHg}$ and $\mathrm{pH}$ at $7.20-7.25$. Rats in the $\mathrm{HH}+\mathrm{NAC}$ group were treated with an intraperitoneal injection of $150 \mathrm{mg} / \mathrm{kg}$ NAC (MedChemExpress, Monmouth, NJ, USA; cat. no. HY-B0215) for $30 \mathrm{~min}$ before being exposed to $5 \% \mathrm{CO}_{2}$ and $16 \% \mathrm{O}_{2}$. The rats, which were used for Western blotting analysis and immunofluorescence staining, were not performed invasive manipulation except ventilation.

Measurement of cerebral oxygen extraction ratio $\left(\mathrm{CERO}_{2}\right)$

The right femoral artery and the right jugular vein was cannulated upstream. The blood samples were collected at $3 \mathrm{~h}$ after ventilation. The hemoglobin concentration $(\mathrm{Hb})$, saturation of artery blood oxygen 
$\left(\mathrm{SaO}_{2}\right), \mathrm{PaO}_{2}$, saturation of jugular vein blood oxygen $\left(\mathrm{SjVO}_{2}\right)$, and pressure of jugular vein blood oxygen $\left(\mathrm{PjVO}_{2}\right)$ were measured using a blood gas/electrolyte analyzer (Model 5700, IL, San Diego, CA, USA). The content of artery blood oxygen $\left(\mathrm{CaO}_{2}\right)$, content of jugular vein blood oxygen $\left(\mathrm{CjVO}_{2}\right)$, and $\mathrm{CERO}_{2}$ were calculated using the following formulas:

[Due to technical limitations, please see the supplementary files section to access the formulas.]

Measurement of the partial pressure of brain tissue oxygen $\left(\mathrm{PbtO}_{2}\right)$

The levels of $\mathrm{PbtO}_{2}$ were measured at $0.5,1,1.5,2,2.5$, and $3 \mathrm{~h}$ after ventilation. To evaluate the $\mathrm{PbtO}_{2}$, a midline incision over vertex was performed after anesthesia. After this, a hole was drilled caudal to the coronal suture, $4 \mathrm{~mm}$ from the midline. The dura was punctured and a microsensor for $\mathrm{PbtO}_{2}$ was inserted into brain tissue[24]. A monitor (Integra CAMO2, Integra LifeSciences Limited, County Offaly, Ireland) was used to measure the $\mathrm{PbtO}_{2}$.

\section{ROS evaluation of brain tissue}

The ROS of brain tissue was evaluated using a ROS ELISA kit (Dogesce, Beijing, China; cat. no. DG21175D) at $3 \mathrm{~h}$ after ventilation following the manufacturers' instructions. Briefly, samples and standards ( $50 \mu \mathrm{l} /$ well) were added to the plate wells coated by antibodies labeled with HRP, which were used to capture ROS. The plate was incubated for $1 \mathrm{~h}$ at $37^{\circ} \mathrm{C}$. After washing completely, substrate A (50 $\mathrm{\mu l} /$ well) and substrate $\mathrm{B}\left(50 \mathrm{\mu l} /\right.$ well) were added to incubate the plate in a dark place for $15 \mathrm{~min}$ at $37^{\circ} \mathrm{C}$. Then the stop buffer was added, and the optical density (OD) was measured spectrophotometrically at a wavelength of $450 \mathrm{~nm}$. The concentrations of ROS in the samples were then determined by comparing the optical density of the samples to the standard curve

\section{$B V-2$ microglial cell cultures and treatment}

BV-2 microglial cells were purchased form CHI Scientific (cat. no. 7-1502), and were cultured and treated as described in our previous study[9]. Briefly, the cells were cultured with DMEM high glucose (Invitrogen Life Technologies Corporation, Carlsbad, CA, USA; cat. no. 8117121) supplemented with 10\% FBS (Invitrogen Life Technologies Corporation; Carlsbad, CA, USA; cat. no. 42F0374K). The microglial cells were randomly divided into five groups: Control group, high concentration of carbon dioxide group (abbreviated HC group), Hypoxia group, Hypoxia + HC group, and Hypoxia + HC + NAC group. Control group was exposed to $5 \% \mathrm{CO}_{2}+20 \% \mathrm{O}_{2}$. $\mathrm{HC}$ group was exposed to $15 \% \mathrm{CO}_{2}+20 \% \mathrm{O}_{2}$ to maintain $\mathrm{pH}$ of the supernatant at $7.20-7.25$. Hypoxia group was exposed to $5 \% \mathrm{CO}_{2}+0.2 \% \mathrm{O}_{2}$ to maintain partial pressure of oxygen $\left(\mathrm{PO}_{2}\right)$ of the supernatant at $55-60 \mathrm{mmHg}$. Hypoxia $+\mathrm{HC}$ group was exposed to $15 \%$ $\mathrm{CO}_{2}+0.2 \% \mathrm{O}_{2}$ to maintain $\mathrm{PO}_{2}$ at $55-60 \mathrm{mmHg}$ and $\mathrm{pH}$ at $7.20-7.25$. The cells in the Hypoxia $+\mathrm{HC}+$ NAC group were treated with 2 mM NAC (MedChemExpress, Monmouth, NJ, USA; cat. no. HY-B0215) for $30 \mathrm{~min}$ before being exposed to $15 \% \mathrm{CO}_{2}+0.2 \% \mathrm{O}_{2}$. 
The OCR was evaluated using a cellulate OCR Assay Kit (BestBio, Shanghai, China; cat. no. BB-48211) after treatment with $0.2 \% \mathrm{O}_{2}$ and $15 \% \mathrm{CO}_{2}$ for $0 \mathrm{~h}, 6 \mathrm{~h}, 12 \mathrm{~h}$, and $24 \mathrm{~h}$. The intervention time and testing time were chosen, when the levels of OCR peaked (In this study, $12 \mathrm{~h}$ was chosen as the intervention time, and 18 min was chosen as the testing time). The OCR was measured following the manufacturers' instructions. Briefly, BV-2 microglial cells were seeded in 96 -well plates (5000 cells/well) and cultured in DMEM high glucose supplemented with $10 \%$ FBS. When the cells spread to $80 \%$ of the bottom of the well, they were treated with different concentrations of $\mathrm{CO}_{2}$ and $\mathrm{O}_{2}$. After the treatment, the medium was changed to DMEM high glucose without FBS, and then fluorescent probes (10 $\mu \mathrm{l} /$ well) were added sequentially. Finally, oxygen mounting medium was added ( 2 drops/well). The OCR levels were examined every three minutes until half an hour with a fluorescent microplate reader (Model 9260, IL-COR ${ }^{\circledR}$ inc, LINCOLN, NE, USA). The excited and emitted wavelength were 485/20 nm and 590/35 nm, respectively.

\section{ROS measurement in microglia}

The ROS production in BV-2 microglial cells was evaluated using a ROS assay kit (BestBio, Shanghai, China; cat. no. BB-4705-2) following the manufacturers' instructions. Briefly, DCFH-DA was diluted with DMEM high glucose without FBS (1: 1500). The coverslips with adherent BV-2 microglial cells were cultured with DMEM high glucose supplemented with $10 \%$ FBS. After the treatment, the medium was changed to diluted DCFH-DA ( $2 \mathrm{ml} /$ well). Then the plates were incubated for $20 \mathrm{~min}$ at $37{ }^{\circ} \mathrm{C}, 5 \% \mathrm{CO}_{2}$. The coverslips were washed with DMEM high glucose without FBS. Finally, the coverslips were mounted by a fluorescent mounting medium and detected using a fluorescence microscope (Olympus DP73 Microscope, Olympus, Tokyo, Japan).

\section{Western blotting analysis.}

Total proteins from the hippocampus tissue and BV-2 microglial cells ( $\mathrm{n}=4$ for each group) were extracted using a Total Protein Extraction Kit (BestBio, Shanghai, China; cat. no. BB-3101-100T). Protein concentrations were determined using a BCA Protein Assay Kit (Invitrogen Life Technologies Corporation, Carlsbad, CA, USA; cat. no. 23227). Equal amounts of protein from each sample were separated in a $15 \%$ SDS-PAGE gel and transferred to PVDF membranes, which were blocked with $5 \%$ non-fat milk for $1 \mathrm{~h}$ at room temperature. After this, the following primary antibodies were added to incubate the membranes overnight at $4{ }^{\circ} \mathrm{C}$ : caspase-1 (1: 1000, Abcam, Cambridge, MA, USA; cat. no. ab1872) and IL-1 $\beta$ (1: 1000, Abcam, Cambridge, MA, USA; Cat. No. ab9722). The membranes were washed on the following day, and HRP-labeled goat anti-rabbit antibody (1: 3000; Cell Signaling Technology; cat. no. 7074S) was added to incubate the membranes for $2 \mathrm{~h}$ at $4{ }^{\circ} \mathrm{C}$. The immunoblots were visualized using a chemiluminescence kit (Bioworld Technology, St. Louis Park, MN, USA; cat. no. AC36131), and detected by an imaging densitometer (ImageQuant LAS 500, GE Healthcare Bio-Sciences AB, Uppsala, Sweden). The relative density was quantified using FluorChem 8900 software (version 4.0.1, Alpha Innotech Corporation, San Leandro CA, USA). $\beta$-actin was used as the control. 
In vivo, the rats were anesthetized with pentobarbital sodium and transcardially perfused with normal saline and $4 \%$ paraformaldehyde at $3 \mathrm{~h}$ after ventilation. The brains were harvested and post-fixed in $4 \%$ paraformaldehyde. This tissue was then dehydrated in graded sucrose and cut into sections of $10 \mu \mathrm{m}$ thickness. The sections were blocked in $5 \%$ normal donkey serum for $0.5 \mathrm{~h}$ at room temperature. In vitro, the coverslips with adherent BV-2 microglial cells were fixed with $4 \%$ paraformaldehyde at $24 \mathrm{~h}$ after treatment. The coverslips were blocked in $5 \%$ normal donkey serum for $0.5 \mathrm{~h}$ at room temperature sequentially.

After that, the following primary antibodies were added to incubate the sections/coverslips overnight at 4 'C: caspase-1 (1: 100; Abcam, Cambridge, MA, USA; cat. no. ab1872), IL-1ß (1: 100; Abcam, Cambridge, MA, USA; cat. no. ab9722), and Iba1 (1: 100; Abcam, Cambridge, MA, USA; cat. no. ab15690). The sections/coverslips were washed on the following day, and the secondary antibodies Alexa Fluor ${ }^{\circledR} 549$ Goat Anti-Rabbit IgG ( $\mathrm{H}+\mathrm{L})$ (1:100; Invitrogen Life Technologies, Carlsbad, CA, USA; cat. no. ATRJN1301) and Alexa Fluor ${ }^{\circledR} 488$ Goat anti-mouse lgG (1:100; Invitrogen Life Technologies, Carlsbad, CA, USA; cat. no. ATRMR2301) were added to incubate the sections/coverslips for $1 \mathrm{~h}$ at room temperature. Finally, the sections/coverslips were mounted by the fluorescent mounting medium with DAPI (Sigma, St. Louis, MO, USA; cat. no. SLBW4468) and detected using a fluorescence microscope (Olympus DP73 Microscope, Olympus, Tokyo, Japan).

\section{Statistical analysis}

The statistical analysis was performed by the SPSS19.0 statistical (IBM, New York, USA). All values are expressed as mean \pm standard deviation. Repeated measures one-way analysis of variance (ANOVA) was used to analyze the repeated measurement data. Factorial ANOVA was for the interaction effects. When an interaction was examined, simple effects analyses were evaluated. Differences were considered statistically significant if the $P$ value $<0.05$.

\section{Results}

Hypercapnia increased cerebral oxygen extraction rate $\left(\mathrm{CERO}_{2}\right)$ in hypoxemic rats

Significant interaction effects were observed between hypercapnia treatment and hypoxia treatment $(P<$ 0.01) (Fig. 1a). Simple effects analyses found an increased $\mathrm{CERO}_{2}$ in Hypoxemia group $(P<0.05)$, but not in Hypercapnia group $(P>0.05)$ compared with Sham group. $\mathrm{HH}$ group had the highest $\mathrm{CERO}_{2}$ levels as compared with Hypoxemia group $(P<0.01)$ and Hypercapnia group $(P<0.01)$ (Fig. 1b).

Hypercapnia decreased partial pressure of brain tissue oxygen $\left(\mathrm{PbtO}_{2}\right)$ in hypoxemic rats

Compared with the Sham group, decreased $\mathrm{PbtO}_{2}$ levels were found in Hypoxemia group $(0.5 \mathrm{~h}-3 \mathrm{~h}: P<$ 0.01), but not in Hypercapnia group (0.5 h - 3 h: $P>0.05$ ) (Fig. 2b, c). HH group had higher $\mathrm{PbtO}_{2}$ levels as 
compared with Hypoxemia group in the first 1.5 hours $(0.5 \mathrm{~h}: P<0.01 ; 1 \mathrm{~h}: P<0.01 ; 1.5 \mathrm{~h}: P<0.05)$ (Fig. $2 \mathrm{~b})$. On the contrary, $\mathrm{HH}$ group had lower $\mathrm{PbtO}_{2}$ levels as compared with Hypoxemia group after 2 hours (2.5 h: $P<0.05 ; 3$ h: $P<0.05)$ (Fig. 2c).

\section{Hypercapnia induced overproduction of ROS in hypoxemic hippocampus}

Significant interaction effects were observed between hypercapnia treatment and hypoxia treatment $(P<$ 0.05) (Fig. 3a). Simple effects analyses found increased levels of ROS in Hypoxemia group $(P<0.01)$, but not in Hypercapnia group $(P>0.05)$ compared with Sham group. HH group had the highest ROS levels as compared with Hypoxemia group $(P<0.01)$ and Hypercapnia group $(P<0.01)$ (Fig. 3b).

Hypercapnia enhanced NLRP3 inflammasome activation via inducing ROS overproduction in microglia in the hypoxic hippocampus

Significant interaction effects were observed between hypercapnia treatment and hypoxia treatment $(P<$ 0.01) (Fig. 4b). Simple effects analyses found increased protein expression levels of caspase -1 in Hypoxemia group $(P<0.01)$, but not in Hypercapnia group $(P>0.05)$ compared with Sham group. $\mathrm{HH}$ group had the highest expression levels of caspase- 1 as compared with Hypoxemia group $(P<0.01)$ and Hypercapnia group $(P<0.01)$ (Fig. 4c). Additionally, the protein expression of caspase- 1 was significantly suppressed with NAC pretreatment $(150 \mathrm{mg} / \mathrm{kg})$ in rats $(P<0.01)$ (Fig. 4c). Double immunofluorescence was used to examine caspase- 1 expression in microglia of hippocampus (Fig. 4 d). Enhanced caspase- 1 immunofluorescence was observed in Hypoxemia group, but not in Hypercapnia group compared with Sham group. $\mathrm{HH}$ group had the most intense caspase-1 fluorescence when compared with Hypoxemia group and Hypercapnia group. In rats given NAC treatment, caspase-1 fluorescence was noticeably attenuated (Fig. 4d).

Hypercapnia increased IL $-1 \beta$ expression via inducing ROS overproduction in microglia in the hypoxic hippocampus

Significant interaction effects were observed between hypercapnia treatment and hypoxia treatment $(P<$ 0.01 ) (Fig. 5b). Simple effects analyses found increased protein expression levels of IL $-1 \beta$ in Hypoxemia group $(P<0.01)$, but not in Hypercapnia group $(P>0.05)$ compared with Sham group. HH group showed the highest expression levels of IL-1 $\beta$ in comparison with Hypoxemia group $(P<0.01)$ and Hypercapnia group $(P<0.01)$ (Fig. 5c). Additionally, the protein expression of IL $-1 \beta$ was significantly suppressed with NAC pretreatment $(150 \mathrm{mg} / \mathrm{kg})$ in rats $(P<0.01)$ (Fig. $5 \mathrm{c})$. Double immunofluorescence was used to examine IL $-1 \beta$ expression in microglia of hippocampus (Fig. $5 d$ ). Enhanced IL $-1 \beta$ immunofluorescence was observed in Hypoxemia group, but not in Hypercapnia group compared with Sham group. HH group had the strongest IL $-1 \beta$ fluorescence as compared with Hypoxemia group and Hypercapnia group. IL-1 $\beta$ fluorescence was evidently reduced in rats given NAC pretreatment (Fig. 5d).

$15 \% \mathrm{CO}_{2}$ increased the oxygen consumption rate (OCR) in hypoxic BV-2 microglial cells 
The OCR levels of BV-2 microglial cells were examined after the treatment with $0.2 \% \mathrm{O}_{2}$ and $15 \% \mathrm{CO}_{2}$ for $0 \mathrm{~h}, 6 \mathrm{~h}, 12 \mathrm{~h}$, and $24 \mathrm{~h}$. The OCR levels were the highest in $12 \mathrm{~h}$ group as compared with other groups (12 h vs 0 h: $P<0.01 ; 12$ h vs 6 h: $P>0.05 ; 12$ h vs 24 h: $P<0.01)$. Besides, the OCR levels were increased time-dependently and achieved a stationary phase at $18 \mathrm{~min}$ (Fig. 6a). In view of this, microglia was treated for 12 hours, and the OCR was examined at $18 \mathrm{~min}$ after the treatment (Fig. 6b, C). Significant interaction effects were observed between $0.2 \% \mathrm{O}_{2}$ treatment and $15 \% \mathrm{CO}_{2}$ treatment $(P<0.01)$ (Fig. 6b). Simple effects analyses found increased levels of OCR in Hypoxia group $(P<0.01)$, but not in HC group $(P>0.05)$ compared with Control group. Hypoxia $+\mathrm{HC}$ group had the highest levels of OCR as compared with Hypoxia group $(P<0.01)$ and $\mathrm{HC}$ group $(P<0.01)$ (Fig. 6C).

\section{$15 \% \mathrm{CO}_{2}$ induced overproduction of ROS in hypoxic $\mathrm{BV}-2$ microglial cells}

Enhanced ROS immunofluorescence was observed in Hypoxia group, but not in $\mathrm{HC}$ group compared with Control group. Hypoxia + HC group had the strongest ROS fluorescence as compared with Hypoxia group and $\mathrm{HC}$ group. ROS fluorescence was obviously reduced with NAC pretreatment (2 mM) in BV-2 microglial cells (Fig. 7).

$15 \% \mathrm{CO}_{2}$ enhanced NLRP3 inflammasome activation via inducing ROS overproduction in hypoxic BV-2 microglial cells

Significant interaction effects were observed between $0.2 \% \mathrm{O}_{2}$ treatment and $15 \% \mathrm{CO}_{2}$ treatment $(P<$ 0.01) (Fig. 8b). Simple effects analyses found increased protein expression levels of caspase -1 in Hypoxia group $(P<0.01)$, but not in $\mathrm{HC}$ group $(P>0.05)$ compared with Control group. Hypoxia $+\mathrm{HC}$ group had the highest expression levels of caspase-1 as compared with Hypoxia group $(P<0.01)$ and HC group $(P<0.01)$ (Fig. 8c). Additionally, the protein expression of caspase-1 was significantly suppressed with NAC pretreatment $(2 \mathrm{mM})$ in BV-2 microglial cells $(P<0.01)$ (Fig. $8 \mathrm{c})$. Double immunofluorescence was used to examine caspase- 1 expression in BV-2 microglial cells (Fig. 8d). Enhanced caspase-1 immunofluorescence was observed in Hypoxia group, but not in HC group compared with Control group. Hypoxia $+\mathrm{HC}$ group had the strongest caspase- 1 fluorescence as compared with Hypoxia group and $\mathrm{HC}$ group. Of note, caspase- 1 fluorescence was evidently reduced with NAC pretreatment (2 mM) in BV-2 microglial cells (Fig. 8d).

$15 \% \mathrm{CO}_{2}$ increased IL-1 $\beta$ expression via inducing ROS overproduction in hypoxic BV-2 microglial cells

Significant interaction effects were observed between $0.2 \% \mathrm{O}_{2}$ treatment and $15 \% \mathrm{CO}_{2}$ treatment $(P<$ 0.01 ) (Fig. 9b). Simple effects analyses found increased protein expression levels of IL-1 $\beta$ in Hypoxia group $(P<0.01)$, but not in $\mathrm{HC}$ group $(P>0.05)$ compared with Control group. Hypoxia $+\mathrm{HC}$ group had the highest expression levels of IL $-1 \beta$ as compared with Hypoxia group $(P<0.01)$ and $\mathrm{HC}$ group $(P<$ 0.01) (Fig. 9c). Additionally, the protein expression of IL-1 $\beta$ was significantly suppressed with NAC pretreatment $(2 \mathrm{mM})$ in BV-2 microglial cells $(P<0.01)$ (Fig. 9c). Double immunofluorescence confirmed $\mathrm{IL}-1 \beta$ expression in BV-2 microglial cells (Fig. 9d). Enhanced IL $-1 \beta$ immunofluorescence was observed 
in Hypoxia group, but not in $\mathrm{HC}$ group compared with Control group. Hypoxia + HC group had the strongest IL $-1 \beta$ fluorescence as compared with Hypoxia group and HC group. IL-1 $1 \beta$ fluorescence was markedly suppressed with NAC pretreatment ( $2 \mathrm{mM}$ ) in BV-2 microglial cells (Fig. 9d).

\section{Discussion}

The present results have shown that hypercapnia enhanced the NLRP3 inflammasome activation via inducing ROS overproduction in hypoxia-activated microglia in vitro and in vivo. This was evident by the increased $\mathrm{CERO}_{2}$, decreased $\mathrm{PbtO}_{2}$, and increased expression levels of caspase- 1 and IL-1 $\beta$ in hypoxiaactivated microglia cells.

The present rat and cell models of hypercapnia/hypoxemia were established as described in our previous study. In the models, $\mathrm{PO}_{2}$ levels of the arterial blood and culture supernatant were maintained at 55-60 $\mathrm{mmHg}$ and $\mathrm{pH}$ levels of the arterial blood and culture supernatant at 7.20-7.25[9]. These are consistent with the change of hypoxemia and permissive hypercapnia in ARDS[25-28].

We reporeted in our previous study that hypercapnia-induced NLRP3 inflammasome activation in hypoxia-activated microglia could upregulate the expression of IL-1 $\beta$. It is well documented that IL-1 $\beta$ promotes a cascade of inflammatory response in the central nervous system that can lead to the aggravation of neuronal injury[9]. Under hypoxic conditions, ROS is known to be critical for NLRP3 inflammasome activation[29-31]. Thus, the expression levels of caspase-1 and IL-1 $\beta$ in the microglia in this study were determined, and the production of ROS in the hippocampus was also evaluated. Significant interaction effects were observed on ROS production, caspase- 1 and IL $-1 \beta$ expression between hypercapnia treatment and hypoxia treatment. More importantly, we have shown that hypercapnia upregulated the expression of caspase -1 and IL $-1 \beta$ in hypoxic hippocampus microglia via inducing ROS overproduction. Remarkably, caspase -1 and IL-1 expression in hypoxic hippocampus microglia was downregulated when ROS was scavenged by NAC. These results indicate that hypercapnia enhanced the NLRP3 inflammasome activation via inducing ROS overproduction.

To determine the effect of hypercapnia on oxidative metabolism of the rat brain, cerebral oxygen extraction ratio $\left(\mathrm{CERO}_{2}\right)$ and partial pressure of brain tissue oxygen $\left(\mathrm{PbtO}_{2}\right)$ were examined. Hypoxemia, as a typical symptom of ARDS, decreases oxidative metabolism rate of the brain to avoid or reduce cerebral damage via preventing the $\mathrm{PbtO}_{2}$ from dropping to a very low level[23]. In this study, we showed hypercapnia alone was not enough to increase or decrease the levels of $\mathrm{PbtO}_{2}$ and $\mathrm{CERO}_{2}$. In the first 1.5 hours, the levels of $\mathrm{PbtO}_{2}$ were elevated by hypercapnia in the hypoxemic rats. This may be the reason why hypercapnia was neuroprotective in rats with transient global cerebral ischemia-reperfusion injury[32] and lateral fluid percussion injury[33]. However, hypercapnia significantly decreased the levels of $\mathrm{PbtO}_{2}$ in the hypoxemic rats after 2 hours' ventilation. It was further evidenced by the increased levels of $\mathrm{CERO}_{2}$ at $3 \mathrm{~h}$ after ventilation. These results suggest that hypercapnia is neuroprotective within a very 
short period of time $(1.5 \mathrm{~h})$, but hypercapnia could aggravate neuronal functions injury over a protracted period via increasing $\mathrm{CERO}_{2}$ and decreasing $\mathrm{PbtO}_{2}$.

In vitro, to determine the effect of a high concentration of $\mathrm{CO}_{2}$ on oxidative metabolism in hypoxic $\mathrm{BV}-2$ microglial cells, the oxygen consumption rate (OCR) was evaluated. There was an interaction effect on the levels of OCR between $0.2 \% \mathrm{O}_{2}$ treatment and $15 \% \mathrm{CO}_{2}$ treatment. The high concentration of $\mathrm{CO}_{2}$ treatment could increase the levels of OCR in hypoxic BV-2 microglial cells, which were consistent with that hypercapnia increased levels of $\mathrm{CERO}_{2}$ in hypoxic rats. The results suggest that hypercapnia could intensify microglial hypoxia both in vivo and in vitro.

To ascertain if a high concentration of $\mathrm{CO}_{2}$ would activate NLRP3 inflammasome via inducing ROS overproduction in hypoxia-activated BV-2 microglial cells, the production of ROS and expression of caspase -1 and $\mathrm{IL}-1 \beta$ was determined. The results were consistent with in vivo experiments. Significant interaction effects were observed on ROS production, caspase- 1 and IL-1 $1 \beta$ expression between $0.2 \% \mathrm{O}_{2}$ treatment and $15 \% \mathrm{CO}_{2}$ treatment. The high concentration of $\mathrm{CO}_{2}$ upregulated the expression of caspase -1 and IL-1 $\beta$ in hypoxia-activated microglia via inducing ROS overproduction, as evidenced by the downregulated expression of caspase -1 and IL $-1 \beta$ when ROS was scavenged. All in all, the results support that a high concentration of $\mathrm{CO}_{2}$ enhanced the NLRP3 inflammasome activation in hypoxiaactivated BV-2 microglial cells via inducing and augmenting ROS overproduction.

In summary, the present results have demonstrated the underlying mechanism whereby hypercapnia can enhance the activation of NLRP3 inflammasome in hypoxic microglia. In this connection, hypercapnia was found to intensify the cerebral hypoxia via increasing $\mathrm{CERO}_{2}$ and decreasing $\mathrm{PbtO}_{2}$. As a consequence, ROS was overproduced in hypoxic microglial cells coupled with activation of NLRP3 inflammasome. Remarkedly, caspase -1 and IL $-1 \beta$ expression was doenregulated when ROS was scavenged. Thus, hypercapnia-induced ROS overproduction and NLRP3 inflammasome activation in microglia may be a potential target to mitigate neuronal damage in the central nervous system such as that in the hippocampus.

\section{Conclusions}

Hypercapnia-induced ROS overproduction via intensifying cerebral hypoxia may activate the NLRP3 inflammasome and enhance $\mathrm{IL}-1 \beta$ release in hypoxia-activated microglia.

\section{Abbreviations}

ARDS: acute respiratory distress syndrome; CNS: central nervous system; IL-1ß: interleukin-1 beta; NLRP3: NLR family, pyrin domain-containing 3; $\mathrm{PbtO}_{2}$ : partial pressure of brain tissue oxygen; OCR: oxygen consumption rate; ROS: reactive oxygen species; $\mathrm{Hb}$ : hemoglobin concentration; $\mathrm{PCO}_{2}$ : partial pressure of carbon dioxide; $\mathrm{PaO}_{2}$ : partial pressure of artery blood oxygen; $\mathrm{SaO}_{2}$ : saturation of artery blood 
oxygen; $\mathrm{PjVO}_{2}$ : pressure of jugular vein blood oxygen; $\mathrm{CaO}_{2}$ : content of artery blood oxygen; $\mathrm{CjVO}_{2}$ : content of jugular vein blood oxygen; $\mathrm{SjVO}_{2}$ : saturation of jugular vein blood oxygen

\section{Declarations}

Ethics approval and consent to participate

The Experimental Animal Care and Use Committee at Jinan University approved all animal procedure protocols (No. 20171011001), which conform to the Guide for the Care and Use of Laboratory Animals published by the US National Institutes of Health. All efforts were made to minimize the numbers of animals used and ensure minimal suffering.

Consent for publication

Not applicable

Availability of data and materials

The datasets used and analyzed during the current study are available from the corresponding author on reasonable request.

Competing interests

The authors declare that they have no competing interests.

\section{Funding}

This work was supported by the Natural Science Foundation of Guangdong province (2016A030311043 and 2017A030313691) and the Medical Scientific Research Foundation of Guangdong Province (A2019135).

\section{Authors' contributions}

DHG participated in making the animal model, assessed IL $-1 \beta$ and caspase -1 expression in the hypoxic hippocampus microglia, collected data, and drafted the manuscript. LXS carried out the measurement of cerebral oxygen extraction ratio and partial pressure of brain tissue oxygen. LXQ conducted BV-2 microglial cells culture and treatment. WKR participated in evaluating ROS production of brain tissue and BV-2 microglial cells. LY participated in the evaluation of oxygen consumption rate evaluation in vitro. WMY performed statistical analysis. ZHK carried out the design of the study. All authors read and approved the final manuscript.

\section{Acknowledgments}

The authors would like to thank Mr. ZhengKang Ding and Mrs. ZiXi Yang for technical assistance. 
${ }^{1}$ Department of Emergency and Critical Care Medicine, Guangdong Provincial People's Hospital and Guangdong Academy of Medical Sciences, 106 ZhongshanEr Road, Guangzhou 510080, China.

${ }^{2}$ Southern Medical University, 1838 North Guangzhou Avenue, Guangzhou 510515, China. ${ }^{3}$ School of Medicine, South China University of Technology, Guangzhou Higher Education Mega Center, Guangzhou 510006, China

\section{References}

1. Papazian L, Aubron C, Brochard L, Chiche JD, Combes A, Dreyfuss D, et al. Formal guidelines: management of acute respiratory distress syndrome. Ann Intensive Care. 2019;9:69.

2. Matthay MA, Zemans RL, Zimmerman GA, Arabi YM, Beitler JR, Mercat A, et al. Acute respiratory distress syndrome. Nat Rev Dis Primers. 2019;5:18.

3. Duan EH, Adhikari N, D'Aragon F, Cook DJ, Mehta S, Alhazzani W, et al. Management of Acute Respiratory Distress Syndrome and Refractory Hypoxemia. A Multicenter Observational Study. Ann Am Thorac Soc. 2017;14:1818-1826.

4. Meade MO, Young D, Hanna S, Zhou Q, Bachman TE, Bollen C, et al. Severity of Hypoxemia and Effect of High-Frequency Oscillatory Ventilation in Acute Respiratory Distress Syndrome. Am J Respir Crit Care Med. 2017;196:727-733.

5. Shen Y, Cai G, Gong S, Dong L, Yan J, Cai W. Interaction between low tidal volume ventilation strategy and severity of acute respiratory distress syndrome: a retrospective cohort study. Crit Care. 2019;23:254.

6. Fielding-Singh V, Matthay MA, Calfee CS. Beyond Low Tidal Volume Ventilation: Treatment Adjuncts for Severe Respiratory Failure in Acute Respiratory Distress Syndrome. Crit Care Med. 2018;46:18201831.

7. Brower RG, Matthay MA, Morris A, Schoenfeld D, Thompson BT, Wheeler A. Ventilation with lower tidal volumes as compared with traditional tidal volumes for acute lung injury and the acute respiratory distress syndrome. N Engl J Med. 2000;342:1301-1308.

8. Barnes T, Zochios V, Parhar K. Re-examining Permissive Hypercapnia in ARDS: A Narrative Review. Chest. 2018;154:185-195.

9. Ding HG, Deng YY, Yang RQ, Wang QS, Jiang WQ, Han YL, et al. Hypercapnia induces IL-1beta overproduction via activation of NLRP3 inflammasome: implication in cognitive impairment in hypoxemic adult rats. J Neuroinflammation. 2018;15:4.

10. Liu HD, Li W, Chen ZR, Hu YC, Zhang DD, Shen W, et al. Expression of the NLRP3 inflammasome in cerebral cortex after traumatic brain injury in a rat model. Neurochem Res. 2013;38:2072-2083.

11. Zhang N, Zhang X, Liu X, Wang H, Xue J, Yu J, et al. Chrysophanol inhibits NALP3 inflammasome activation and ameliorates cerebral ischemia/reperfusion in mice. Mediators Inflamm. 2014;2014:370530. 
12. Gustin A, Kirchmeyer M, Koncina E, Felten P, Losciuto S, Heurtaux T, et al. NLRP3 Inflammasome Is Expressed and Functional in Mouse Brain Microglia but Not in Astrocytes. PLoS One. 2015;10:e130624.

13. Mangan M, Olhava EJ, Roush WR, Seidel HM, Glick GD, Latz E. Targeting the NLRP3 inflammasome in inflammatory diseases. Nat Rev Drug Discov. 2018;17(8):588-606.

14. Swanson KV, Deng M, Ting JP. The NLRP3 inflammasome: molecular activation and regulation to therapeutics. Nat Rev Immunol. 2019;19(8):477-89.

15. He Y, Hara H, Nunez G. Mechanism and Regulation of NLRP3 Inflammasome Activation. Trends Biochem Sci. 2016;41(12):1012-21.

16. Kelley N, Jeltema D, Duan Y, He Y. The NLRP3 Inflammasome: An Overview of Mechanisms of Activation and Regulation. Int J Mol Sci. 2019;20.

17. Hamilton C, Anand PK. Right place, right time: localisation and assembly of the NLRP3 inflammasome. F1000Res. 2019;8.

18. Fann DY, Lee SY, Manzanero S, Chunduri P, Sobey CG, Arumugam TV. Pathogenesis of acute stroke and the role of inflammasomes. Ageing Res Rev. 2013;12:941-966.

19. Lai M, Yao H, Shah S, Wu W, Wang D, Zhao Y, et al. The NLRP3-Caspase 1 Inflammasome Negatively Regulates Autophagy via TLR4-TRIF in Prion Peptide-Infected Microglia. Front Aging Neurosci. 2018;10:116.

20. Murphy MP. How mitochondria produce reactive oxygen species. Biochem J. 2009;417:1-13.

21. Fuhrmann DC, Brune B. Mitochondrial composition and function under the control of hypoxia. Redox Biol. 2017;12:208-215.

22. Hernansanz-Agustin P, Ramos E, Navarro E, Parada E, Sanchez-Lopez N, Pelaez-Aguado L, et al. Mitochondrial complex I deactivation is related to superoxide production in acute hypoxia. Redox Biol. 2017;12:1040-1051.

23. Watts ME, Pocock R, Claudianos C. Brain Energy and Oxygen Metabolism: Emerging Role in Normal Function and Disease. Front Mol Neurosci. 2018;11:216.

24. Wang CC, Kuo JR, Chen YC, Chio CC, Wang JJ, Lin BS. Brain tissue oxygen evaluation by wireless near-infrared spectroscopy. J Surg Res. 2016;200:669-675.

25. Briva A, Lecuona E, Sznajder Jl. Permissive and non-permissive hypercapnia: mechanisms of action and consequences of high carbon dioxide levels. Arch Bronconeumol. 2010;46:378-382.

26. Thorens JB, Jolliet P, Ritz M, Chevrolet JC. Effects of rapid permissive hypercapnia on hemodynamics, gas exchange, and oxygen transport and consumption during mechanical ventilation for the acute respiratory distress syndrome. Intensive Care Med. 1996;22:182-191.

27. Hickling KG, Walsh J, Henderson S, Jackson R. Low mortality rate in adult respiratory distress syndrome using low-volume, pressure-limited ventilation with permissive hypercapnia: a prospective study. Crit Care Med. 1994;22:1568-1578. 
28. Dushianthan A, Cusack R, Chee N, Dunn JO, Grocott MP. Perceptions of diagnosis and management of patients with acute respiratory distress syndrome: a survey of United Kingdom intensive care physicians. BMC Anesthesiol. 2014;14:87.

29. Yu X, Lan P, Hou X, Han Q, Lu N, Li T, et al. HBV inhibits LPS-induced NLRP3 inflammasome activation and IL-1 beta production via suppressing the NF-kappaB pathway and ROS production. $J$ Hepatol. 2017;66(4):693-702.

30. Han Y, Xu X, Tang C, Gao P, Chen X, Xiong X, et al. Reactive oxygen species promote tubular injury in diabetic nephropathy: The role of the mitochondrial ros-txnip-nlrp3 biological axis. Redox Biol. 2018;16:32-46.

31. Tschopp J, Schroder K. NLRP3 inflammasome activation: The convergence of multiple signalling pathways on ROS production? Nat Rev Immunol. 2010;10:210-215.

32. Zhou Q, Cao B, Niu L, Cui X, Yu H, Liu J, et al. Effects of permissive hypercapnia on transient global cerebral ischemia-reperfusion injury in rats. Anesthesiology. 2010;112:288-297.

33. Yang WC, Wang Q, Chi LT, Wang YZ, Cao HL, Li WZ. Therapeutic hypercapnia reduces blood-brain barrier damage possibly via protein kinase Cepsilon in rats with lateral fluid percussion injury. $\mathrm{J}$ Neuroinflammation. 2019;16:36.

\section{Figures}

a

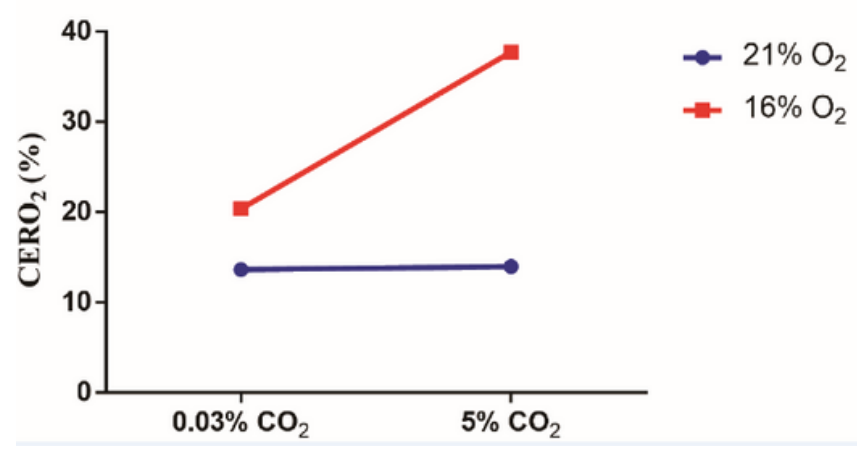

b

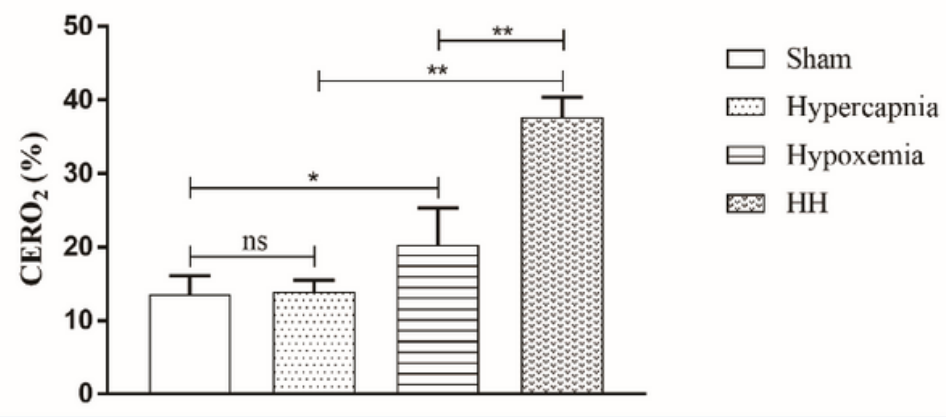

\section{Figure 1}

Hypercapnia increased CERO2 in hypoxemic rats $(n=4)$. a There is an interaction effect between hypoxia treatment and hypercapnia treatment $(P<0.01)$. $b$ Simple effects analyses show an increased CERO2 in Hypoxemia group ( ${ }^{*}<0.05$ ), but not in Hypercapnia group (ns $P>0.05$ ) compared with Sham group. $\mathrm{HH}$ group has the highest CERO2 levels as compared with Hypoxemia group ( $* * P<0.01)$ and Hypercapnia group ( $* \star \mathrm{P}<0.01$ ). ns, non-significant; Sham group, sham-operated group; HH group, hypercapnia + hypoxemia group; CERO2, cerebral oxygen extraction rate. The concentrations of 02 and CO2 in the air are $21 \%$ and $0.03 \%$. 
a

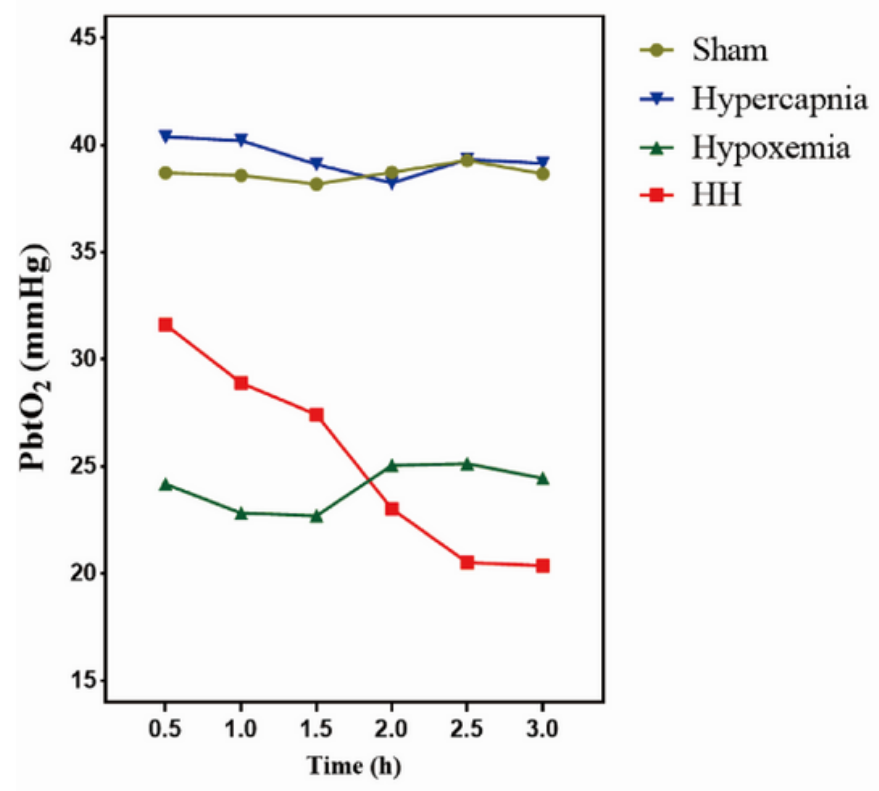

b

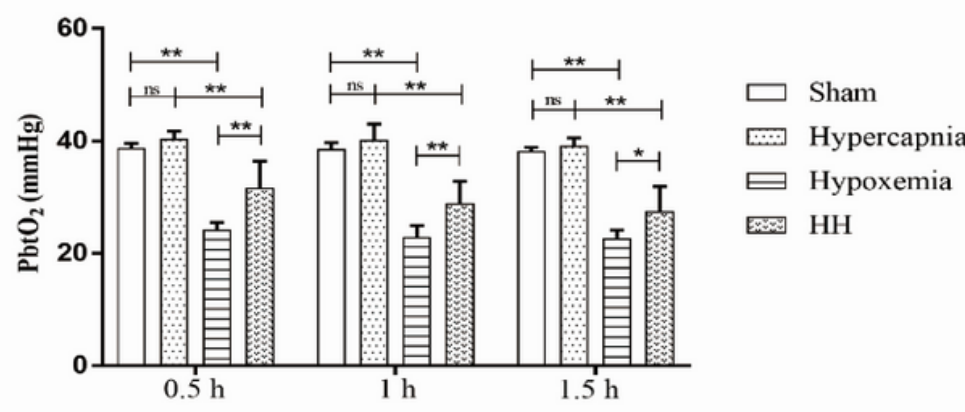

C

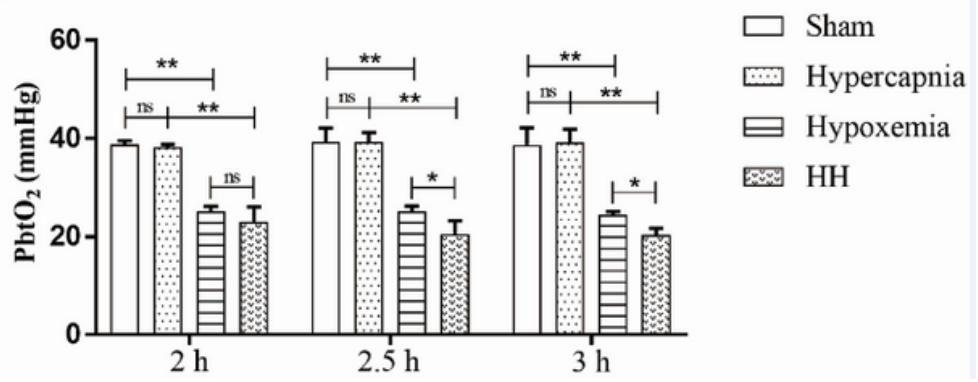

Figure 2

Hypercapnia decreased Pbt02 in hypoxemic rats $(n=4)$. Graphs a illustrate the PbtO2 at 0.5, 1, 1.5, 2, 2.5, and $3 \mathrm{~h}$ after ventilation in the rats. b, c Compared with the Sham group, Pbt02 levels are decreased in Hypoxemia group $(0.5 \mathrm{~h}-3 \mathrm{~h}: \mathrm{P}<0.01)$, but not in Hypercapnia group $(0.5 \mathrm{~h}-3 \mathrm{~h}: \mathrm{P}>0.05)$. HH group has higher Pbt02 levels as compared with Hypoxemia group in the first 1.5 hours $(0.5 \mathrm{~h}: \mathrm{P}<0.01 ; 1 \mathrm{~h}: \mathrm{P}<$ $0.01 ; 1.5 \mathrm{~h}$ : $\mathrm{P}<0.05)$. On the contrary, $\mathrm{HH}$ group has lower $\mathrm{PbtO} 2$ levels as compared with Hypoxemia group after 2 hours $(2.5$ h: $\mathrm{P}<0.05 ; 3$ h: $\mathrm{P}<0.05)$. Sham group, sham-operated group; HH group, hypercapnia + hypoxemia group; Pbt02, partial pressure of brain tissue oxygen.

a

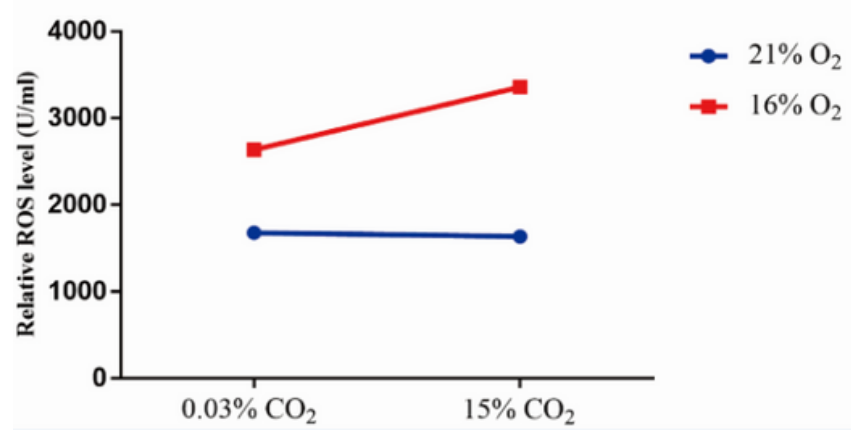

b

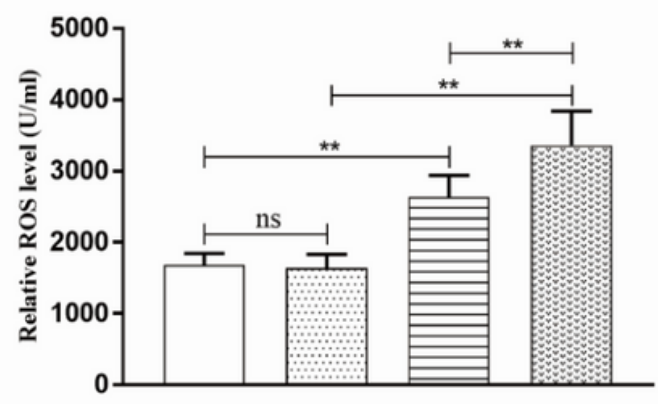

$\square$ Sham

Hypercapnia

$\boxminus$ Hypoxemia

하워 $\mathrm{HH}$

\section{Figure 3}

Hypercapnia induced overproduction of ROS in hypoxemic hippocampus $(n=4)$. a There is an interaction effect between hypoxia treatment and hypercapnia treatment $(P<0.05)$. b Simple effects analyses show increased levels of ROS in Hypoxemia group ( $* \star P<0.01$ ), but not in Hypercapnia group (ns $P>0.05$ ) compared with Sham group. $\mathrm{HH}$ group has the highest ROS levels as compared with Hypoxemia group 
(** $\mathrm{P}<0.01$ ) and Hypercapnia group (** $\mathrm{P}<0.01)$. ns, non-significant; Sham group, sham-operated group; $\mathrm{HH}$ group, hypercapnia + hypoxemia group; ROS, reactive oxygen species. The concentrations of 02 and CO2 in the air are $21 \%$ and $0.03 \%$.

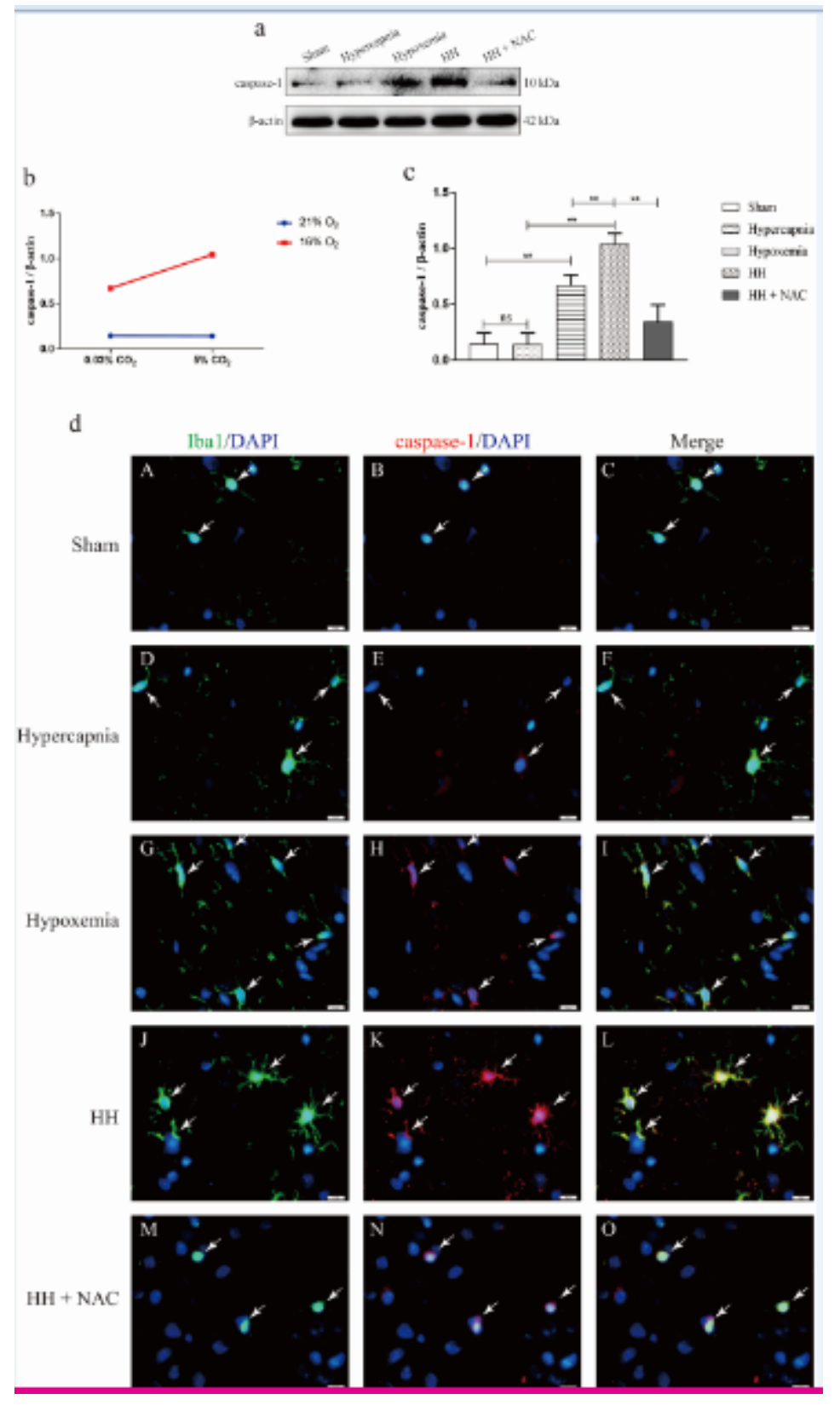

Figure 4

Hypercapnia enhanced NLRP3 inflammasome activation via inducing ROS overproduction in hypoxic microglia ( $n=4)$. a Immunoreactive bands of caspase-1 (10 kDa) and $\beta$-actin $(42 \mathrm{kDa})$. b There is an interaction effect between hypoxia treatment and hypercapnia treatment $(P<0.01)$. c Simple effects analyses show increased protein expression levels of caspase- 1 in Hypoxemia group ( $* \star P<0.01$ ), but not in Hypercapnia group ( $\mathrm{ns} \mathrm{P}>0.05$ ) compared with Sham group. $\mathrm{HH}$ group has the highest expression levels of caspase-1 as compared with Hypoxemia group ( $* * \mathrm{P}<0.01)$ and Hypercapnia group $(* \star \mathrm{P}<$ 0.01). The protein expression of caspase-1 is significantly suppressed with NAC pretreatment (150 $\mathrm{mg} / \mathrm{kg})$ in rats $(* \star \mathrm{P}<0.01)$. $\mathrm{d}$ Immunofluorescence images showing the expression of Iba1+ microglia (A, 
$D, G, J, M$, green), caspase-1 (B, E, H, K, N, red), and the co-localization of caspase-1 and microglia ( $C, F, I$, $L, 0$ ). Enhanced caspase-1 immunofluorescence is evident in Hypoxemia group, but not in Hypercapnia group compared with Sham group. HH group emits the strongest caspase-1 fluorescence as compared with Hypoxemia group and Hypercapnia group. Caspase-1 fluorescence is markedly attenuated in rats given NAC pretreatment. Scale bars: $10 \mu \mathrm{m}$. ns, non-significant; Sham group, sham-operated group; $\mathrm{HH}$ group, hypercapnia + hypoxemia group. The concentrations of 02 and $\mathrm{CO} 2$ in the air are $21 \%$ and $0.03 \%$.

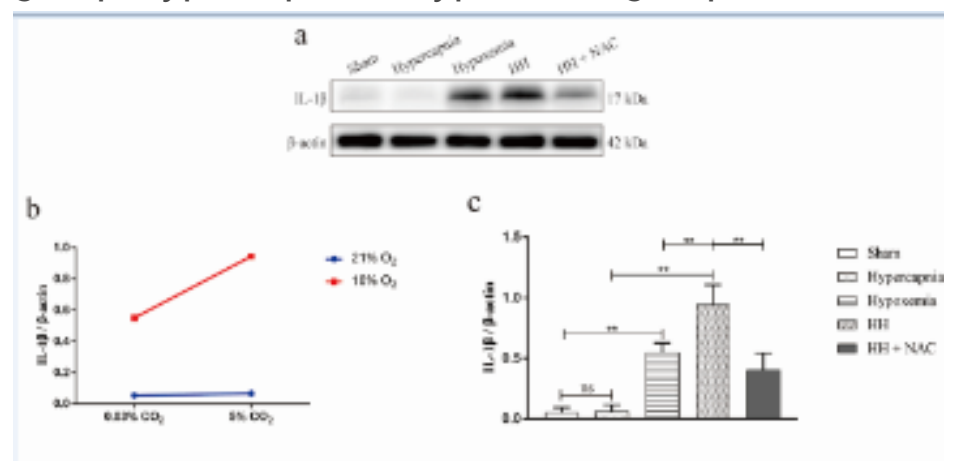

d
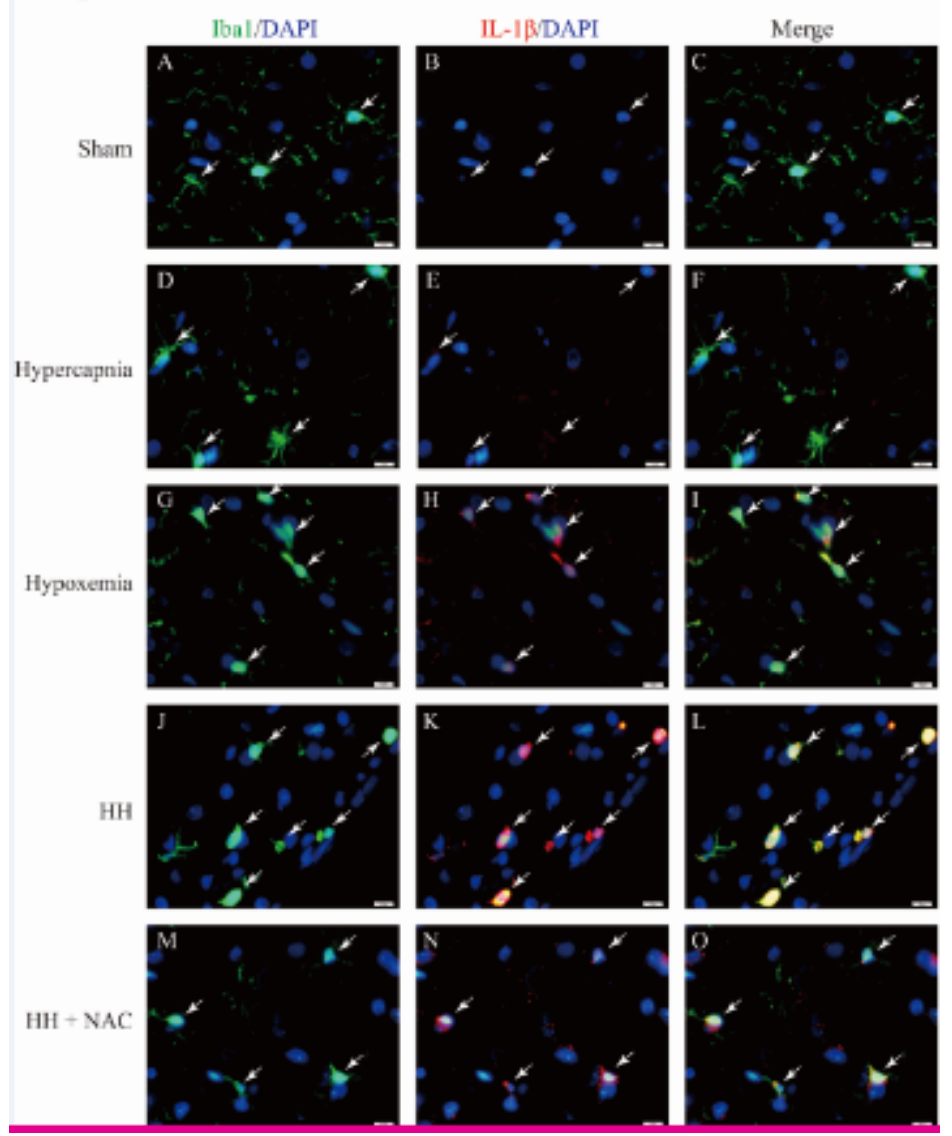

\section{Figure 5}

Hypercapnia increased IL-1 $\beta$ expression via inducing ROS overproduction in hypoxic microglia $(n=4)$. a Immunoreactive bands of IL-1 $\beta$ ( $17 \mathrm{kDa})$ and $\beta$-actin $(42 \mathrm{kDa})$. b There is an interaction effect between hypoxia treatment and hypercapnia treatment $(P<0.01)$. c Simple effects analyses show increased protein expression levels of IL-1 $\beta$ in Hypoxemia group ( $* \star P<0.01$ ), but not in Hypercapnia group (ns $P>$ 0.05) compared with Sham group. HH group has the highest expression levels of IL-1 $\beta$ as compared with 
Hypoxemia group ( $* \star P<0.01$ ) and Hypercapnia group (** $P<0.01)$. The protein expression of IL-1 $\beta$ is significantly suppressed with NAC pretreatment $(150 \mathrm{mg} / \mathrm{kg})$ in rats $(* \star P<0.01)$. d Immunofluorescence images showing the expression of Iba $1+\operatorname{microglia}(A, D, G, J, M$, green), IL-1 $\beta(B, E, H, K, N$, red), and the co-localization of IL-1 $\beta$ and microglia (C, F, I, L, O). Enhanced IL-1 $\beta$ immunofluorescence is evident in Hypoxemia group, but not in Hypercapnia group compared with Sham group. $\mathrm{HH}$ group has the strongest IL-1 $\beta$ fluorescence as compared with Hypoxemia group and Hypercapnia group. IL-1 $\beta$ fluorescence is markedly reduced in rats given NAC pretreatment. Scale bars: $10 \mu \mathrm{m}$. IL-1 $\beta$, interleukin-1 beta; ns, nonsignificant; Sham group, sham-operated group; HH group, hypercapnia + hypoxemia group. The concentrations of 02 and $\mathrm{CO} 2$ in the air are $21 \%$ and $0.03 \%$.

a

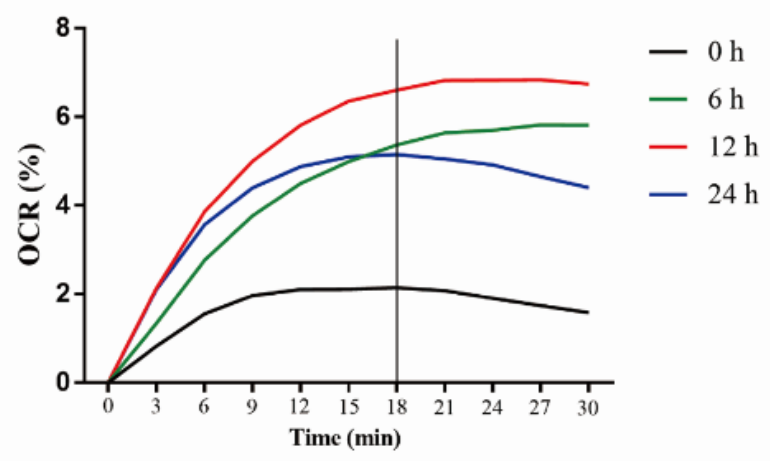

b

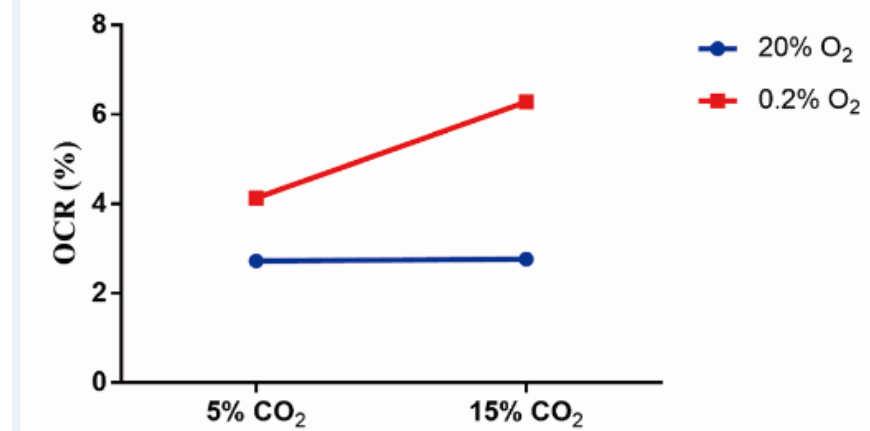

c

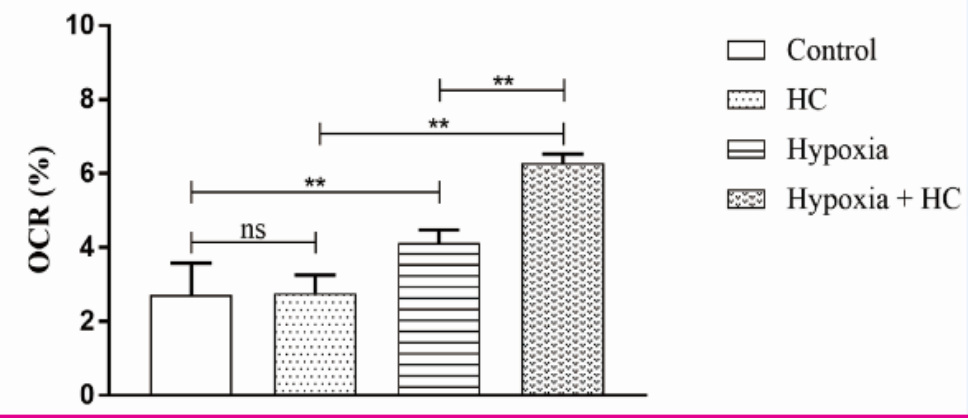

Figure 6

$15 \%$ CO2 increased the OCR in hypoxic BV-2 cells $(n=4)$. a The OCR levels are the highest in $12 \mathrm{~h}$ group as compared with other groups ( $12 \mathrm{~h}$ vs $0 \mathrm{~h}$ : $\mathrm{P}<0.01 ; 12 \mathrm{~h}$ vs $6 \mathrm{~h}: \mathrm{P}>0.05 ; 12 \mathrm{~h}$ vs $24 \mathrm{~h}$ : $\mathrm{P}<0.01)$. The OCR levels are increased time-dependently and achieved a stationary phase at $18 \mathrm{~min}$. So the microglia cells were treated for 12 hours, and the OCR was examined at 18 min after the treatment, as shown in graph $b$ and c. b There is an interaction effect between $0.2 \% 02$ treatment and $15 \%$ CO2 treatment $(P<$ 0.01). c Simple effects analyses show increased levels of OCR in Hypoxia group ( $* \star P<0.01$ ), but not in $\mathrm{HC}$ group (ns $\mathrm{P}>0.05$ ) compared with Control group. Hypoxia $+\mathrm{HC}$ group has the highest levels of OCR as compared with Hypoxia group ( $* \star P<0.01$ ) and $\mathrm{HC}$ group ( $* \star \mathrm{P}<0.01)$. ns, non-significant; HC group, high concentration of carbon dioxide group. 

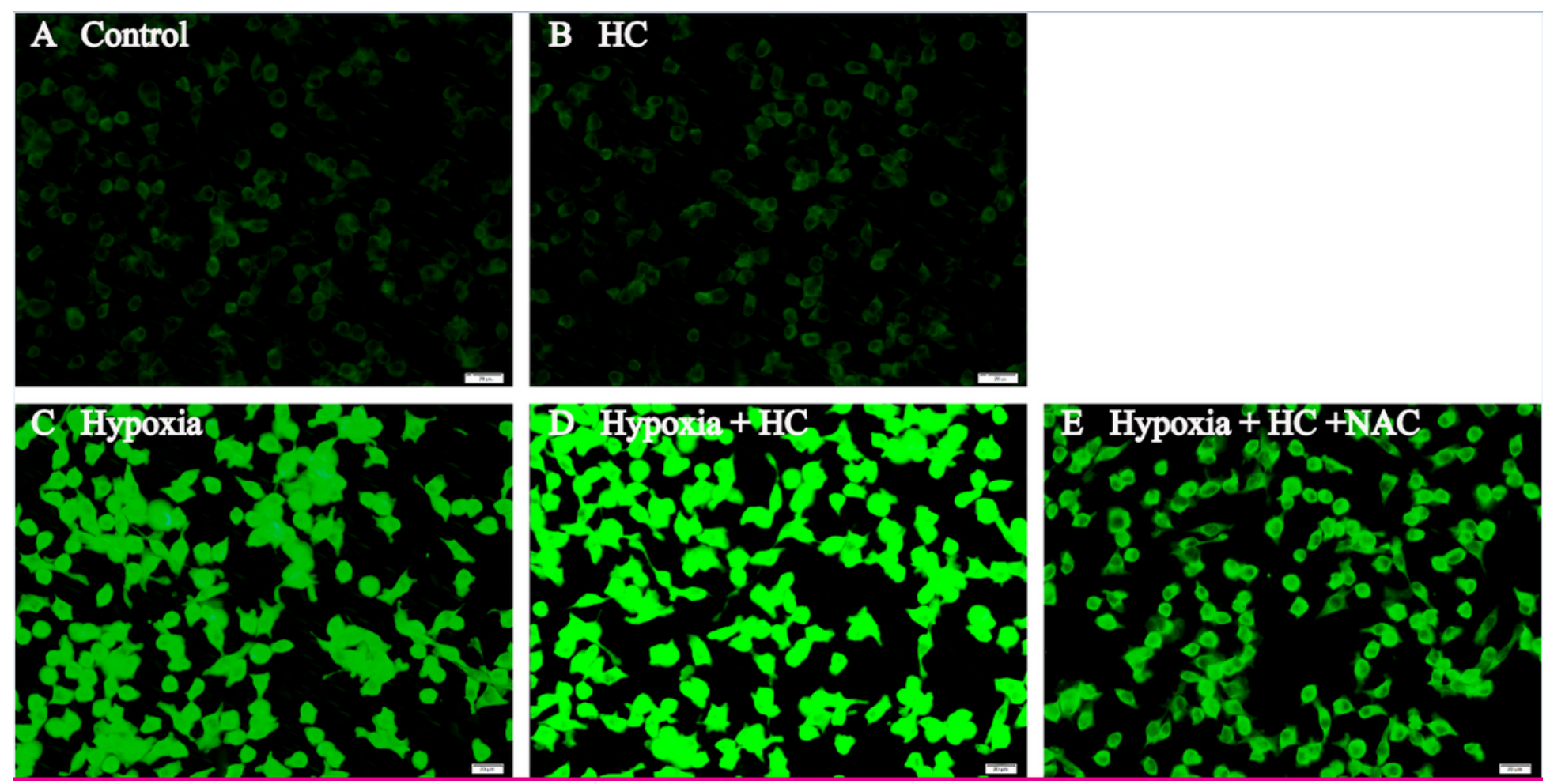

Figure 7

$15 \%$ CO2 induced overproduction of ROS in hypoxic BV-2 cells $(n=4)$. Immunofluorescence images showing the production of ROS (A, B, C, D, E, green). Enhanced ROS immunofluorescence is evident in Hypoxia group, but not in $\mathrm{HC}$ group compared with Control group. Hypoxia $+\mathrm{HC}$ group exhibits the most intense ROS fluorescence as compared with Hypoxia group and HC group. ROS fluorescence is noticeably reduced with NAC pretreatment ( $2 \mathrm{mM}$ ) in BV-2 microglial cells. Scale bars: $20 \mu \mathrm{m}$. ROS, reactive oxygen species; $\mathrm{HC}$ group, high concentration of carbon dioxide group. 


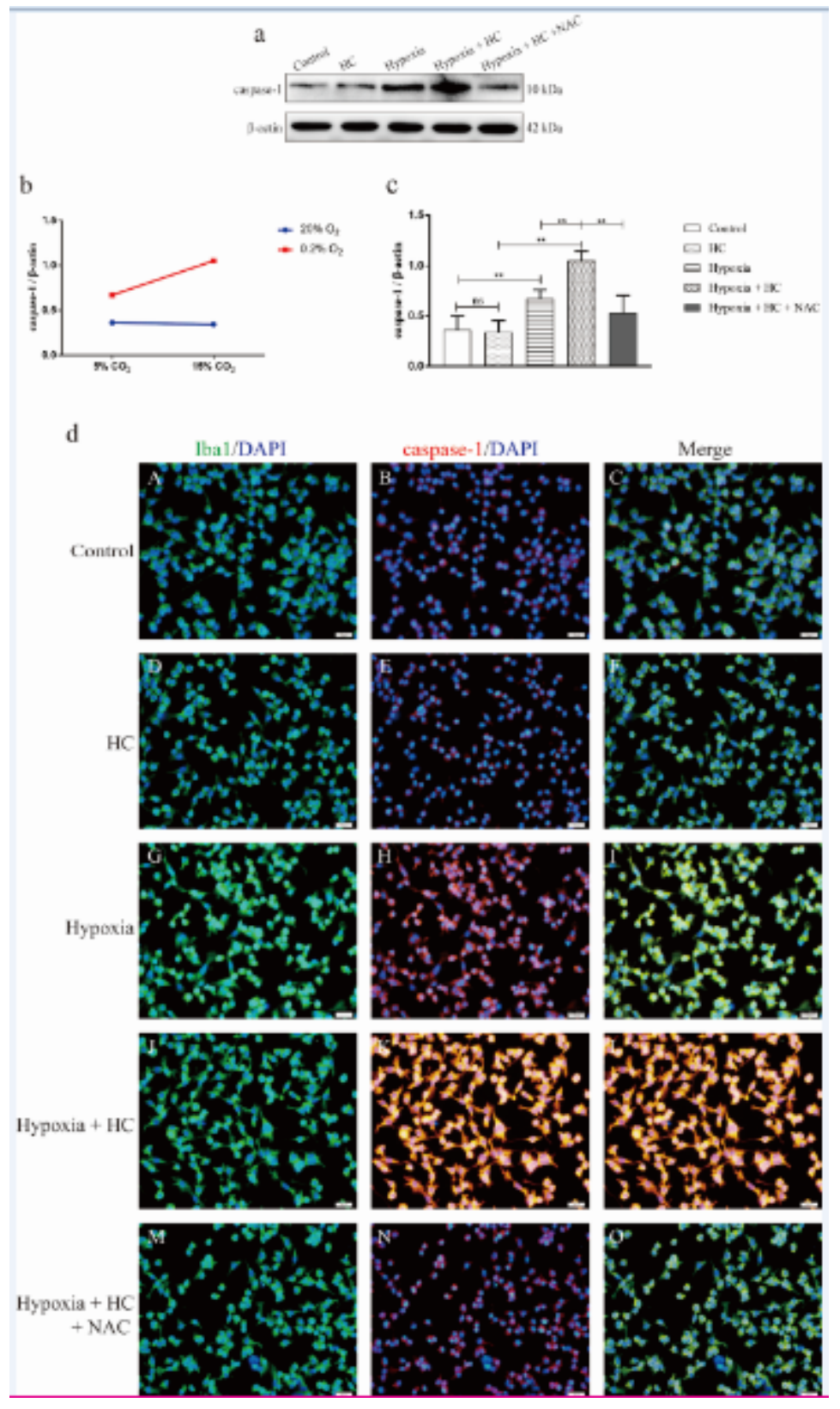

Figure 8

15\% CO2 enhanced NLRP3 inflammasome activation via inducing ROS overproduction in hypoxic BV-2 cells. a Immunoreactive bands of caspase- $1(10 \mathrm{kDa})$ and $\beta$-actin $(42 \mathrm{kDa})$. b There is an interaction effect between $0.2 \% 02$ treatment and $15 \% \mathrm{CO} 2$ treatment $(P<0.01, n=4)$. c Simple effects analyses show increased protein expression levels of caspase-1 in Hypoxia group ( $* * P<0.01$ ), but not in $\mathrm{HC}$ group (ns $\mathrm{P}>0.05$ ) compared with Control group. Hypoxia $+\mathrm{HC}$ group shows the highest expression levels of caspase-1 in comparion with Hypoxia group ( ${ }^{\star *} \mathrm{P}<0.01$ ) and $\mathrm{HC}$ group ( $\left.{ }^{\star \star} \mathrm{P}<0.01\right)$. Additionally, the protein expression of caspase- 1 is significantly suppressed with NAC pretreatment (2 mM) in BV-2 microglial cells ( $* * P<0.01$ ). $d$ Immunofluorescence images showing the expression of Iba1+ microglia (A, D, G, J, M, green), caspase-1 (B, E, H, K, N, red), and the co-localization of caspase-1 and microglia (C, F, $\mathrm{I}, \mathrm{L}, \mathrm{O}$ ). Enhanced caspase-1 immunofluorescence is evident in Hypoxia group, but not in $\mathrm{HC}$ group compared with Control group. Hypoxia + HC group shows the strongest caspase- 1 fluorescence as 
compared with Hypoxia group and HC group. Caspase-1 fluorescence is drastically reduced with NAC pretreatment $(2 \mathrm{mM})$ in BV-2 microglial cells. Scale bars: $10 \mu \mathrm{m}$. ns, non-significant; HC group, high concentration of carbon dioxide group.

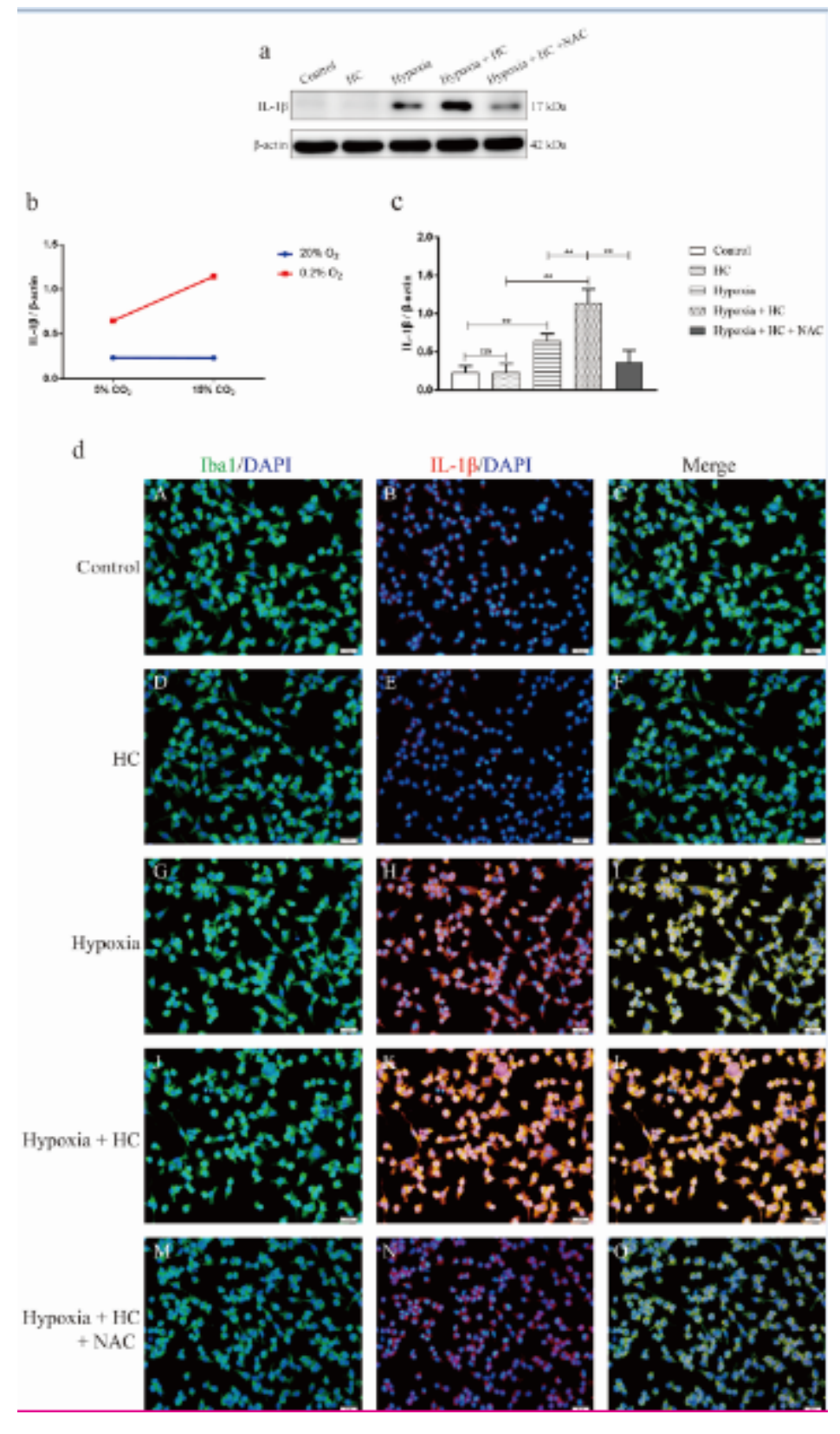

Figure 9

$15 \%$ CO2 increased IL-1 $\beta$ expression via inducing ROS overproduction in hypoxic BV-2 cells. a Immunoreactive bands of IL-1 $\beta$ (17 kDa) and $\beta$-actin (42 kDa). b There is an interaction effect between $0.2 \% 02$ treatment and $15 \%$ CO2 treatment $(P<0.01, n=4)$. c Simple effects analyses show increased protein expression levels of IL-1 $\beta$ in Hypoxia group ( $* * P<0.01$ ), but not in $\mathrm{HC}$ group (ns $\mathrm{P}>0.05$ ) compared with Control group. Hypoxia $+\mathrm{HC}$ group shows the highest expression levels of IL-1 $\beta$ as compared with Hypoxia group ( $* * \mathrm{P}<0.01$ ) and HC group (** $\mathrm{P}<0.01)$ (Fig. 9c). Additionally, the protein expression of IL-1 $\beta$ is significantly suppressed with NAC pretreatment $(2 \mathrm{mM})$ in BV-2 microglial cells (** $P<0.01)$. $d$ Immunofluorescence images showing Iba1+ microglia ( $A, D, G, J, M$, green), IL-1 $\beta$ labeling (B, 
$E, H, K, N$, red), and their co-localization ( $C, F, I, L, O)$. Enhanced IL-1 $\beta$ immunofluorescence is evident in Hypoxia group, but not in $\mathrm{HC}$ group compared with Control group. Hypoxia $+\mathrm{HC}$ group displays the strongest IL-1 $\beta$ fluorescence when compared with Hypoxia group and HC group. IL-1 $\beta$ fluorescence is markedly reduced with NAC pretreatment $(2 \mathrm{mM})$ in BV-2 microglial cells. Scale bars: $10 \mu \mathrm{m}$. IL-1ß, interleukin-1 beta; ns, non-significant; $\mathrm{HC}$ group, high concentration of carbon dioxide group.

\section{Supplementary Files}

This is a list of supplementary files associated with this preprint. Click to download.

- Methodsformulas.docx 